\title{
DISCONTINUOUS FUNCTIONS WITH THE DARBOUX PROPERTY
}

\author{
Israel Halperin \\ (received October 16,1958 )
}

1. Introduction. If $f(x)$ is real-valued and continuous, it has the property that it takes on all intermediate values when it passes from one value to another. This means that whenever $f\left(x_{1}\right)$ and $f\left(x_{2}\right)$ are different and $u$ is any number between them, then $f(x)=u$ for at least one $x$ between $x_{1}$ and $x_{2}$. We shall call this the Darboux property.

Until the work of Darboux in 1875 some mathematicians believed that this property actually implied continuity of $f(x)$. Darboux showed that there are discontinuous functions with the property of Darboux. He proved the theorem, which we shall call Darboux's theorem, that this property holds for every derivative function and he constructed a derivative function which is discontinuous for all rational $x$. 1)

Now how badly behaved can a function be and still have the Darboux property? Darboux's function is Riemann-integrable but later Volterra gave a derivative function which is not Riemann-integrable. 2) Then Lebesgue gave a function with the Darboux property which is not a derivative function, and is discontinuous for all $x .3)$ More recently, the writer constructed functions $f(x)$ which have the Darboux property but which are not Lebesgue measurable on any interval, and for which the equation

1) G. Darboux, Mémoire sur les fonctions discontinues, Annales Scientifiques de l'Ecole Normale Supérieure, 2e série, 4 (1875), 57-112.

2) V. Volterra, Giornale de Battaglini, 1881.

3) H. Lebesgue, Leçons sur l'intégration, (Paris, 1904).

Can. Math. Bull. vol. 2, no. 2, May 1959 
$f(x)=u$ has, for every real $u$, a continuum number of solutions in every interval, indeed in every perfect set ${ }^{4}$ ) (necessary and sufficient conditions for the Darboux property have been given by Csaszar ${ }^{5)}$ ).

This note reviews and comments on the examples given by Darboux, Volterra, Lebesgue and the writer. In what follows the value zero is assigned by convention to the symbols $\sin \left(\frac{1}{0}\right)$ and $\cos \left(\frac{1}{0}\right)$.

2. The Example of Darboux. If the series $\sum_{n=1}^{\infty} f_{n}(x)$ converges uniformly and the $f_{n}(x)$ are all derivative functions, then the sum function is also a derivative function. A proof of this theorem which does not assume Riemann-integrability is given by Lebesgue. ${ }^{6)}$

Now consider the function $\varphi(y)=y^{2} \sin (1 / y)$. The derivative $\varphi^{\prime}(y)=2 y \sin (1 / y)-\cos (1 / y)$ is defined for all y and has a single point of discontinuity, namely $y=0$. Darboux's construction 7) is to set $f(x)=\sum_{n=1}^{\infty} f_{n}(x)$ where

$$
f_{n}(x)=\frac{a_{n}}{n} \frac{d}{d x}\{\varphi(\sin n \pi x)\}=\pi a_{n}(\cos n \pi x)\left\{\varphi^{\prime}(y)\right\}_{y=\sin n \pi x}
$$

the $a_{n}$ being arbitrary real constants with $\sum_{n=1}^{\infty}\left|a_{n}\right|<\infty$. Since $\left|\varphi^{\prime}(y)\right| \leqslant 3$ for $|y| \leqslant 1$ it follows that $f(x)$ is the sum of a uniformly convergent series of derivative functions. Hence $f(x)$ is itself a derivative function and, by the theorem of Darboux, has the Darboux property.

As for discontinuities, each $f_{n}(x)$ is discontinuous precisely at the points $x=m / n$ where $m$ is an arbitrary integer. Hence, at every irrational $x$, the $f_{n}(x)$ are all continuous and so is their uniformly convergent sum $f(x)$. Darboux states, without proof,

4) Israel Halperin, Discontinuous functions with the Darboux property, American Mathematical Monthly 57 (1950), $539-540$.

5) Israel Halperin, On the Darboux property, Pacific Journal of Mathematics 5 (1955), 703-705.

6) Lebesgue, loc. cit., p. 85.

7) Darboux, loc. cit., p. 109. 
that $f(x)$ is discontinuous at every rational $x$. Let us examine this statement.

Suppose $x_{0}=p / q$ in lowest terms, $q>0$. Then $f_{n}(x)$ is continuous at $x_{0}$ unless $n=m q$ for some integer $m$; if $n=m q$,

$$
\begin{aligned}
& f_{m q}(x)=\pi a_{m q}(\cos m q \pi x)\{2 \sin m q \pi x \sin (1 / \sin m q \pi x)\} \\
& +\pi a_{m q}\left(\cos m q \pi x_{0}-\cos m q \pi x\right) \cos (1 / \sin m q \pi x) \\
& +\pi a_{m q} \cos m q \pi x_{0}\left\{-\cos (1 / \sin m q \pi x)+\cos \left(1 / m q \pi\left(x-x_{0}\right)\right)\right\} \\
& -\pi a_{m q} \cos m q \pi x_{0} \cos \left(1 / m q \pi\left(x-x_{0}\right)\right) .
\end{aligned}
$$

Of these four terms, the first three are continuous at $x_{0}$ and bounded in absolute value by $\left|\mathrm{amq}_{\mathrm{mq}}\right|$; hence

$$
f(x)=\left\{\text { function continuous at } x_{0}\right\}-\pi \sum_{m=1}^{\infty} a_{m q}(-1\}^{m} \cos \left(1 / m q \pi\left(x-x_{0}\right)\right)
$$

Thus $f(x)$ is continuous at $x_{0}$ if and only if

$$
\sum_{m=1}^{\infty} a_{m q}(-1)^{p m} \cos (1 / m t) \rightarrow 0 \text { as } t \rightarrow 0 \text {. }
$$

Now if $\sum_{m=1}^{\infty}\left|b_{m}\right|<\infty$, then, by actual integration,

$$
\int_{r \pi}^{(r+1) \pi}\left|\sum_{m=1}^{N} b_{m} \cos \left(n_{m} u\right)\right| 2 d u=(\pi / 2) \sum_{m=1}^{N}\left|b_{m}\right|^{2}
$$

where $N, r$ are any positive integers and the $n_{m}$ are different integers. Hence

$$
\overline{b d}_{r} \leqslant u \leqslant(r+1) \pi\left|\sum_{m=1}^{N} b_{m} \cos \left(n_{m} u\right)\right| \geqslant \sqrt{\frac{1}{2} \sum_{m=1}^{N}\left|b_{m}\right|^{2}} .
$$

Choosing $n_{m}=N ! / m$ and setting $t=(N !) u$ gives

$\overline{b d}_{r(N !) \pi \leqslant t \leqslant(r+1)(N !) \pi}\left|\sum_{m=1}^{N} b_{m} \cos (t / m)\right| \geqslant \sqrt{\frac{1}{2} \sum_{m=1}^{N}\left|b_{m}\right|^{2}}$. This implies

$$
\overline{\lim }_{t \rightarrow \infty}\left|\sum_{m=1}^{\infty} b_{m} \cos (t / m)\right| \geqslant \sqrt{\frac{1}{2} \sum_{m=1}^{\infty}\left|b_{m}\right|^{2}} .
$$

Thus $\sum_{m=1}^{\infty} b_{m} \cos (1 / m t) \rightarrow 0$ as $t \rightarrow 0$ if and only if all $b_{m}$ are zero. So the $f(x)$ of Darboux is discontinuous at $x_{0}$ if and only if $a_{n} \neq 0$ for at least one $n$ which is a multiple of $q$, and is discontinuous at all rational $x$ if and only if, for every $q$, some $a_{m q} \neq 0$, in particular if all $a_{n} \neq 0$.

A discontinuous derivative function similar to that of Darboux but much easier to study would be the sum of any 
uniformly convergent series of discontinuous derivative functions such that no two of them were discontinuous at the same point. For example, since $\cos (1 / x)$ is a derivative function, in fact the derivative of $\int_{0}^{x} 2 t \sin (1 / t) d t-x^{2} \sin (1 / x)$, it follows that the function

$$
g(x)=\sum_{n=1}^{\infty} n^{-2} \cos \left(1 /\left(x-a_{n}\right)\right)
$$

is a derivative function with discontinuities at the preassigned arbitrary countable set of different points $a_{1}, a_{2}, \ldots$.

3. The example of Volterra. The example of Darboux and the $g(x)$ constructed in the preceding section, are both uniformly convergent series of Riemann-integrable functions, and hence themselves Riemann-integrable. Thus their points of discontinuity are sets of zero Lebesgue measure. Volterra's example of a discontinuous derivative function is not even Riemann-integrable, its discontinuities forming a set of positive Lebesgue measure.

Let $\varepsilon$ be any positive number $\leqslant 1$ and let $P$ be a nowhere dense perfect set whose complementary open set $O$ has Lebesgue measure less than $\varepsilon$. An example of such a set $P$ is this: let $r_{1}, r_{2}, \ldots$ be the rational numbers arranged as a sequence, and let $\eta$ be any positive irrational number $<\varepsilon$. Define $I_{1}$ to be the open interval $\left(\mathrm{r}_{1}-\eta / 4, \mathrm{r}_{1}+\eta / 4\right)$ and by induction define $I_{n}$ to be the empty set if $r_{n}$ is contained in a previously defined $I_{m}(m<n)$, otherwise as the open interval $\left(r_{n}-\delta_{n}, r_{n}+\delta_{n}\right)$ where $\delta_{n}=\frac{1}{2} \min \left[\left|r_{n}-r_{m}\right|-\delta_{m}, m=1,2, \ldots, n-1 ; \eta / 2^{n}\right]$. Choose $O$ to be the set union of all $I_{n}$ and $P$ to be the complement of $O$. ${ }^{8)}$

Now let $\varphi(x)=x^{2} \sin (1 / x)$ and construct a function $F(x)$ as follows: If $x$ is in $P$ let $F(x)=0$; if $x$ is in one of the open intervals, say $(a, b)$, of which $O$ is composed, let $d$ be the maximum number $\leqslant \frac{1}{2}(\mathrm{~b}-\mathrm{a})$ for which $(\mathrm{d} / \mathrm{dx})\{\varphi(\mathrm{x})\}=0$ and define

8) For this type of construction in n-dimensional space, see Hahn and Rosenthal, Set Functions, University of New Mexico Press, 1948, p. 98, Theorem 8.2.8. 


$$
\begin{array}{ll}
F(x)=\varphi(x-a) & \text { for } a<x<a+d \\
F(x)=\varphi(d) & \text { for } a+d \leqslant x \leqslant b-d \\
F(x)=\varphi(b-x) & \text { for } b-d<x<b .
\end{array}
$$

The derivative $F^{\prime}(x)$ exists and satisfies $\left|F^{\prime}(x)\right| \leqslant 3$ for all $x$, it is continuous at every $x$ in $O$, and at every $x$ in $P$ $\varlimsup \overline{\lim } F^{\prime}(x+0)=\varlimsup \lim F^{\prime}(x-0)=1$ and $\underline{\lim } . F^{\prime}(x+0)=\underline{\lim } F^{\prime}(x-0)=-1$. Thus $F^{\prime}(x)$, which is Volterra's function, is a bounded derivative function whose points of discontinuity form a set of positive Lebesgue measure; in fact the points of continuity form a set of arbitrarily small positive Lebesgue measure.

As suggested by Lebesgue, a derivative function can be formed whose points of continuity actually have zero Lebesgue measure. Let $P_{n}, F_{n}(x)$ be respectively a perfect set and Volterra function corresponding to $\varepsilon=1 / \mathrm{n}$, let $P$ be the set union of the $P_{n}$, and define a function $h(x)=\sum_{n=1}^{\infty} a_{n} F_{n}^{\prime}(x)$ where the $a_{n}$ are any real numbers satisfying $\sum_{n=1}^{\infty}\left|a_{n}\right|<\infty$. Then $h(x)$ is the sum of a uniformly convergent series of derivative functions and hence itself a derivative function. As for discontinuities, if $x_{O}$ is in $P$ and $P_{n_{O}}$ is the first $P_{n}$ containing $x_{0}$, then

$$
\begin{aligned}
& h(x)-h\left(x_{0}\right)=\sum_{n=1}^{n_{0}-1} a_{n}\left\{F_{n}^{\prime}(x)-F_{n}^{\prime}\left(x_{0}\right)\right\} \\
& +\sum_{n=n_{0}+1}^{\infty} a_{n}\left\{F_{n}^{\prime}(x)-F_{n}^{\prime}\left(x_{0}\right)\right\}+a_{n_{0}}\left\{F_{n_{0}}^{\prime}(x)-F_{n_{0}}^{\prime}\left(x_{0}\right)\right\} .
\end{aligned}
$$

When $x \rightarrow x_{0}$, the first sum $\rightarrow 0$, the second sum has absolute value $\leqslant \sum_{n=n_{0}+1}^{\infty} 6\left|a_{n}\right|$, and the last term has oscillation $2\left|a_{n_{0}}\right|$. Hence $h(x)$ will be discontinuous at every $x$ in $P$ if $\left|a_{m}\right|>6 \sum_{n=m+1}^{\infty}\left|a_{n}\right|$ for all $m$, for example if $a_{n}=13^{-n}$ for all $\mathrm{n}$; the points of continuity of such an $\mathrm{h}(\mathrm{x})$ will form a set whose Lebesgue measure is less than $1 / n$ for all $n$, hence zero.

4. The example of Lebesgue. Every derivative function $f(x)=(d / d x) F(x)$ is the limit as $n \rightarrow \infty$ of the sequence of continuous functions $n\{F(x+1 / n)-F(x)\}$, and therefore, by Baire's Theorem, must have some points of continuity, indeed the points of continuity must be dense. 9) Lebesgue has given the following

9) See Lebesgue, loc. cit., p. 92. 
example of a function with the Darboux property which has no points of continuity and hence is of course not a derivative function.

Let $\mathbf{x}$ be written as a non-terminating decimal I. $a_{1} a_{2} \ldots a_{n} \ldots$. If the decimal . $a_{1} a_{3} \ldots a_{2 n-1} \ldots$ is not periodic, set $f(x)=0$; if it is periodic and the first period commences with $a_{2 n-1}$, set $f(x)=. a_{2 n^{2}} 2 n+2^{a} 2 n+4 \cdots$. This $f(x)$, which is Lebesgue's function, 10$)$ satisfies $0 \leqslant f(x) \leqslant 1$ for all $x$ and in every interval, no matter how small, takes on every value between 0 and $l$ inclusive. Hence it has the Darboux property but is discontinuous for all $\mathrm{x}$.

If for periodic $a_{1} a_{3} \ldots a_{2 n-1} \ldots \ldots f(x)$ had been defined as.$a_{4 n} a_{4 n+2} \cdots a_{4 n+2} \cdots$ plus the integer $a_{2 n} a_{2 n+2} \cdots a_{4 n-2}$ (respectively, plus the integer $(-1)^{n} a_{2 n} a_{2 n+2} \cdots a_{4 n-2}$ ) the values of $f(x)$, on every interval, would have consisted of all non-negative numbers (respectively, all real numbers).

In each case, the number of solutions of the equation $f(x)=u$ for given $u$ with $0 \leqslant \mathrm{u} \leqslant 1$ (respectively, $0 \leqslant \mathrm{u}<\infty$, respectively, $-\infty<\mathrm{u}<+\infty)$ and $\mathrm{u} \neq 0$, is countably infinite, and countably infinite on every interval no matter how small. Again, the $x$ with $x=I . a_{1} a_{2} a_{3} \ldots$ for any fixed periodic sequence $a_{1} a_{3} a_{5} \ldots$, form a Lebesgue null set. Since there is only a countably infinite set of such periodic sequences, it follows that $f(x)=0$ except on a Lebesgue null set.

5. Further Examples 4) We now construct a function, which, like Lebesgue's $f(x)$, has the Darboux property and is discontinuous for all $x$. But this function will take on every real number as value a continuum number of times on every interval; it will be non-Lebesgue measurable on every measurable set of positive measure, and hence will not coincide with a continuous or even derivative function on any measurable set of positive measure.

10) Lebesgue, 1oc. cit., p. 90. This example is cited in L.M. Graves, The Theory of Functions of Real Variables, McGraw-Hill, 1946, p. 65. Lebesgue uses this example to show that the sum of two functions need not have the Darboux property though each of the functions has it. 
Using the axiom of choice, G. Hamel has constructed a basis for all real numbers. 11) This is a transfinite sequence of continuum many real numbers $a_{1}, a_{2}, \ldots, a_{\alpha}, \ldots(\alpha<\Omega)$ such that every real number $x$ can be expressed in one and only way as $x=\sum_{\alpha} r_{\alpha} a_{\alpha}$ with rational coefficients $r_{\alpha}$ of which only a finite number differ from zero. Such a basis can be rearranged into a two-fold sequence

$$
b_{1}, b_{2}, \ldots, b_{\alpha}, \ldots(\alpha<\Omega) ; c_{1}, c_{2}, \ldots, c_{\alpha}, \ldots(\alpha<\Omega) .
$$

Now define the function $h(x)$ by setting $h(x)=\sum_{\alpha} r_{\alpha} a_{\alpha}$ for $x=\sum_{\alpha} r_{\alpha} b_{\alpha}+\sum_{\beta} s_{\beta} c_{\beta}$. For any interval $(a, b)$ and any $u=\sum_{\alpha} r_{\alpha} a_{\alpha}$ we will have $h(x)=u$ if $x=\sum_{\alpha} r_{\alpha} b_{\alpha}+\sum_{\beta} s_{\beta} c_{\beta}$ for all choices of the $s_{\beta}$; in particular if only one $s_{\beta} \neq 0$, say $s_{\beta_{0}}$, and it is chosen so that $a-\sum_{\alpha} r_{\alpha} b_{\alpha}<s \beta_{0} c_{\beta_{0}}<b-\sum_{\alpha} r_{\alpha} b_{\alpha}$. Hence $h(x)$ will equal $u$ for continuum many $x$ in $(a, b)$.

If $h(x)$ were Lebesgue measurable on any measurable set $E$ of positive Lebesgue measure, then for some number $K$ the inequality $h(x)<K$ would hold on a set of positive measure, and this would imply that $h(x)$ is continuous, since it satisfies the functional relation $h(x+y)=h(x)+h(y)$ for all $x, y$. 12) But $h(x)$ is clearly not continuous, so it cannot be even measurable on any set of positive measure.

Using another construction, we can define $f(x)$ so as to be non-measurable on every set of positive measure and take on every real number as value a continuum number of times not only in every interval but even in every perfect set, including those of zero Lebesgue measure. Let $\Omega$ be the smallest ordinal number for which there are continuum ordinal numbers $\alpha<\Omega$. Then, with the axiom of choice, the real numbers can be arranged as a transfinite sequence $u_{1}, u_{2}, \ldots, u_{\alpha}, \ldots(\alpha<\Omega)$. Since there are continuum many perfect sets, there exists, with

11) G. Hamel, Eine Basis aller Zahlen und die unstetigen Losungen der Funktionalgleichung: $f(x+y)=f(x)+f(y)$, Mathematische Annalen 60 (1905), 459-462.

12) A. Ostrowski, Uber die Funktionalgleichung der Exponentialfunktion und verwandte Funktionalgleichungen, Jahresbericht der Deutschen Mathematiker Vereinigung 38 (1929). 
this $\Omega$, a sequence $P_{1}, P_{2}, \ldots, P_{\alpha}, \ldots(\alpha<\Omega)$ in which each perfect set appears continuum many times. Now for each $\alpha$ define $p_{\alpha}$ by induction on $\alpha$, to be the first $u$ not previously selected which is contained in $P_{\alpha}$. Such a $u$ will exist since, for each $\alpha$, less than continuum u's will have been previously selected and the perfect set $P_{\alpha}$ contains continuum many numbers. This process of selection will select continuum many $\mathrm{p}^{\prime} \mathrm{s}$ from each perfect set $P$ since each $P$ appears continuum many times as a $P_{\alpha}$, and the $p^{\prime}$ s selected from different perfect sets will be mutually exclusive. 13) For each perfect set let its selected $\mathrm{p}^{\prime} \mathrm{s}$ be arranged as a double sequence $\left(\mathrm{x}_{\alpha \beta}\right.$; $\alpha, \beta<\Omega)$ and set $h\left(x_{\alpha \beta}\right)=u_{\alpha}$. This $h(x)$ will clearly take on every real number as value a continuum number of times in every perfect set. If $h(x)$ were measurable on any set of positive measure, there would be a $K$ such that the inequality $h(x)<K$ would hold on a set of positive measure, and hence on some perfect subset of it. This contradicts the fact that $h(x)$ takes on all values on every perfect set so that $h(x)$ cannot be measurable on any set of positive measure.

13) This construction was used by W. Sierpinski and N. Lusin to subdivide an interval into continuum many parts each of exterior Lebesgue measure equal to the length of the interval. See their paper in Comptes Rendus (Paris) 165 (1917), $422-424$.

Queen's University 